\title{
The Internal-Gripping Device of the Manipulator Based on Worm and Worm Gear and Crank-Connecting Rod Drive
}

\author{
Xia Chunfeng \\ Dept. of information and mechanical engineering \\ Suzhou Polytechnic Institute of Agriculture \\ Suzhou, China \\ E-mail: yxcf100@aliyun.com \\ Du Qiu \\ Dept. of mechanical and electric engineering \\ Soochow University \\ Suzhou, China \\ E-mail: $15962257244 @ q q . c o m$
}

\author{
Wang Mingdi* \\ Dept. of mechanical and electric engineering \\ Soochow University \\ Suzhou, China \\ E-mail: wangmingdi@suda.edu.cn \\ Zhong Kangmin \\ Dept. of mechanical and electric engineering \\ Soochow University \\ Suzhou, China \\ E-mail: zkm@suda.edu.cn
}

\begin{abstract}
The working principle of an internal-gripping device of the manipulator based on worm and worm gear and crank-connecting rod drive is introduced. Its mechanical properties and calculation formula is also given out. After the device being driven by gear motor, output torque rotates the worm first and transmits the driving force to the worm; Then the worm rotates the upper and the lower worm gears which are meshed with it separately and the worm gears sway the upper and the lower crank-connecting rods articulated in worm gears; Lastly, two times amplification of the force by the force-amplifier of the upper and the lower crank-connecting rods is realized. The gripper can stick close to the object or the inside surface of workpiece accurately, then grip the object and hold it tightly in balance. The device is with compact structure, easy operation, timesaving and less effort, well flexibility and high greenization.
\end{abstract}

Keywords- manipulator; worm and worm gear; the crankconnecting rod; gripping device; gear motor

\section{INTRODUCTION}

With the development of economy and the improvement of requirements for the degree of automation in all walks of life, the robot technology develops rapidly and meanwhile all kinds of manipulators appear [1-2]. The manipulator also known as automatic hand is an automatic operation device which can model some functions of hands' and arms' actions to grip and carry objects or operate tools according to the fixed program. The manipulator can realize the mechanization and automation of production instead of person's heavy work. The manipulator grips and carries objects or operates tools mainly by the end effector installed on it. The end effector which is installed on the wrist of manipulator is an additional device used to carry out some operations or works[3-4]. The end effector is an important part to grip workpieces or clamp special tools to operate directly, which is one of actuators of manipulator. There are many kinds of end effctors and one manipulator can install different kinds of end effectors to adapt to and finish different works or operation requirements. The usages of end effectors can be divided into moving, machining and measuring. The carrying end effector refers to all kinds of clamping devices and is used to grip or absorb the carried objects [5].

In the modern industry, in the field of production line and dangerous goods handling, it is required to use the manipulator to capture, handle and place items or workpiece. Due to complex tasks and diverse workplaces, in the field, part of gripping, handling and placing for objects or the workpiece still rely on manual, which makes the production or work efficiency can not be improved, and with the labor shortage the material handling cost ratio in the production process will be gradually increased. Thus the mechanization and automation in production process has become a prominent theme. The automation of continuous production process in chemical industry and so on has been mostly solved. However, the production is not continuous in mechanical industry such as processing and assembling, etc. Especially, the carry of feeding and blanking still conducted by labor or special machine in domestic mechanical processing, which is very suitable when the product is single and the capacity is not high. However, using labor or special machine to feed and blank material exposes many deficiencies and weaknesses. For example, (1) the structure of special machine is complex and difficult to maintain, which is not conducive to the production of automated assembly line; (2) It doesn't have the characteristic of flexibility, which is difficult to adapt to product changes and is not conducive to the adjustment of product structure; (3) This method is labor-intensive, inefficiency and more accident-prone; Moreover, the stability of product quality is not good enough when 
adapting artificial feeding, which can't meet the production requirements of large quantities and high quality[6].

Considering of the defects of using labor or special machine to feed and blank material, this article designs an internal-gripping device of the manipulator based on worm and worm gear and crank-connecting rod drive, which realizes the purpose of gripping objects or workpiece automatically and evenly. The worm and worm gear has the advantages of smooth transmission, small shock and noise, gaining larger reduction ratio through only single stage drive and good performances of reinforcement and self-locking [7]. This article gains an internal-gripping device of the manipulator which can realize two times amplification of the force and has good self-locking performance by means of combining worm and worm gear with the crank-connecting rod mechanism in series and arranging them symmetrically. The device solves the problem of labor-intensive and inefficiency. Meanwhile, the internal-gripping device of the manipulator can be installed on all kinds of manipulators to adapt to different changes of products, which is beneficial to adjust the product structures and realize automated production. The device are with the characteristics of compact structure, easy operation, well flexibility, high greenization and the nonlinear change of output force and displacement.

\section{THE WORKING PRINCIPLE AND MECHANICS ANALYSIS}

\section{A. The working principle of the electrical-clamping} device which increases the force by secondary tandem

The worm device can be used to transmit motion and power between the two staggered axis, which can get a great deceleration ratio. Since the worm gear teeth is a continuous helical teeth, it is gradually brought into engagement with the worm wheel teeth and gradually withdrawn and simultaneously meshing engagement of a few more teeth, whereupon the driving is smooth and good. Due to good performance of self-locking worm gear and reliable clamping, it is commonly used to clamp in a clamping mechanism [8]. The crank-connecting rod mechanism can transmit force and change the way of movement and transform the rotary motion of crank to move back and forth in straight line [9]. Utilizing above characteristics of the transmission of worm and worm gear and crank-connecting rod mechanism and combining them, the electrical-clamping device which increases the force by secondary tandem is designed. The schematic diagram is shown in Fig .1. This device consists of gear motor, worm and worm gear, crank-connecting rod and indenter.

The working principle of the electrical-clamping device which increases the force by secondary tandem is that after the device being driven by gear motor, output torque rotates the worm first, then the teeth on the worm mesh the worm gear to rotate it clockwise, the force of the device is amplified for the first time at this moment; Then the rotation of the worm gear swings the crank-connecting rod articulated in it and the connecting rod moves downward in a straight line, the force of the device is amplified for the second time at this moment; Lastly, the indenter outputs clamping force and the workpiece is clamped. If the gear motor is stopped now, the whole clamping device will be self-locked. After the workpiece being processed, the gear motor is access to the reverse power supply and the worm rotates the worm gear counterclockwise, then the connecting rod articulated in worm gear moves upward in a straight line to loosen the workpiece.

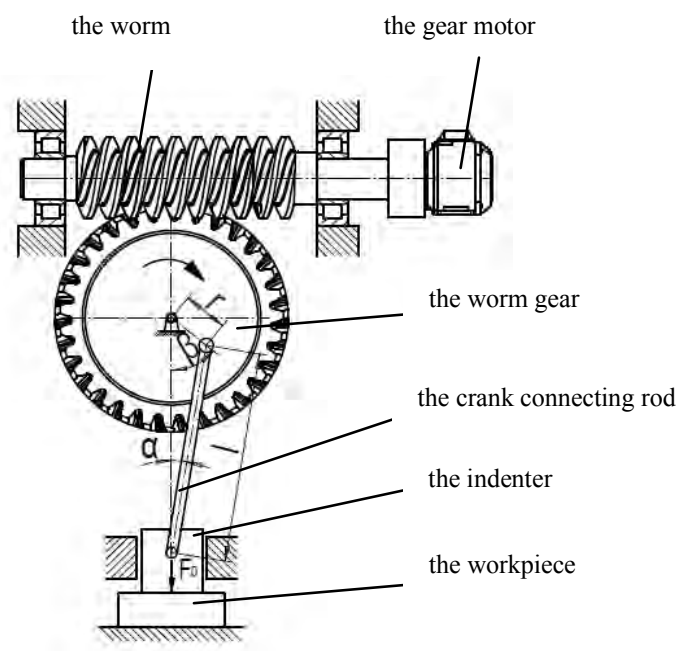

Figure 1. The schematic diagram of the electrical-clamping device which increases the force by secondary tandem

\section{B. The working principle of the internal-gripping device of the manipulator}

By using symmetrical relationship, the electricalclamping device which increases the force by secondary tandem shown in Fig .1 can be evolved to the internalgripping device of the manipulator, shown as Fig .2.

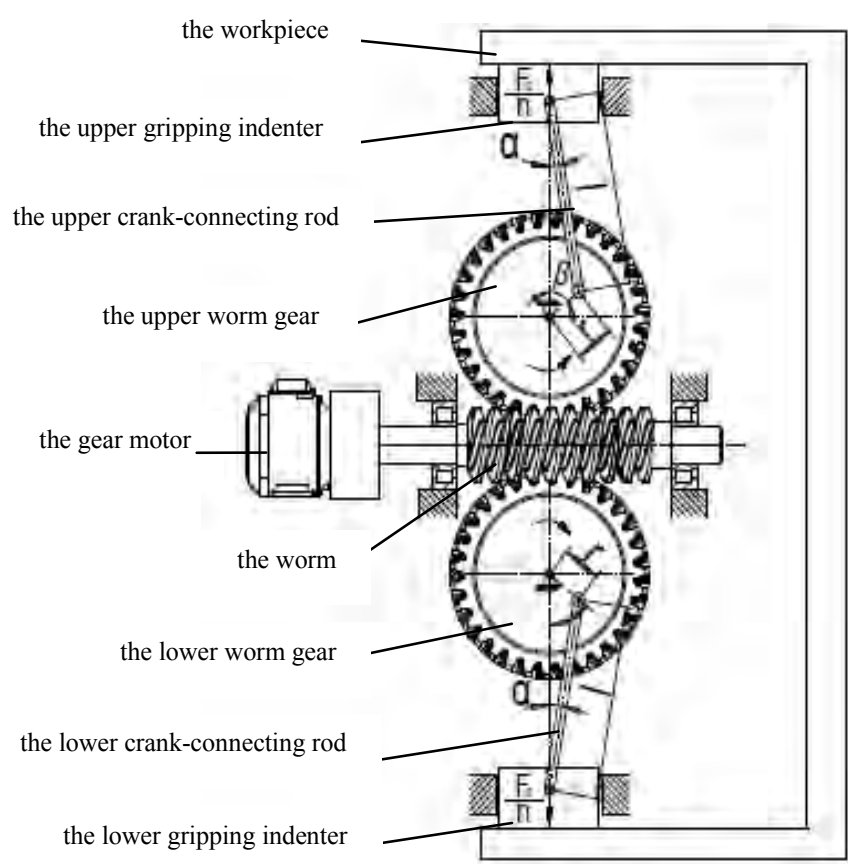

Figure 2. The schematic diagram of the internal-gripping device of the manipulator

This device mainly consists of gear motor, worm, the upper and the lower worm gears, the upper and the lower 
crank-connecting rods and gripping indenters. The worm's position should be fixed both in axial direction and circumferential direction to ensure the stability of transmission. Besides, the upper and the lower worm gears and crank-connecting rods should also be calibrated symmetrically. The gear motor is the motor through reducer, which output low speed and high torque without the need for any intermediate link through a power to convey the precise mechanism, is commonly used for reduction drive prime mover piece of work between machines, which is a green and pollution-free transmission technology [10]. The flexibility of crank and connecting rod mechanism's direction is very good, which can change the output force's position easily according to the actual clamping requirements.

The working principle of the internal-gripping device is that after the device being driven by gear motor, the worm is rotated by output torque, then the upper part of the worm meshes the upper worm gear to rotate it counterclockwise and the lower part of the worm meshes the lower worm gear to rotate it clockwise; The force of the device is amplified for the first time at this moment. After that, the upper and the lower worm gears swing the upper and the lower crank-connecting rods articulated in the worm gears to make the upper connecting rod move upward in a straight line and the lower connecting rod move downward in a straight line, then the gripping indenters get close to the inside surface of the workpiece separately; The force of the device is amplified for the second time by utilizing the angle effects of the upper and lower crank-connecting rods at this moment. Lastly, the output force of the upper and the lower gripping indenters act on the inside surface of the workpiece separately and complete the clamping and gripping movement. If the gear motor is stopped now, the whole gripping device will be self-locked, which means that the gripping force can be kept the same and the workpiece can be hold tightly throughout the whole process of the workpiece's being carried and moved; Also, the device will not go on consuming energy, which can further realize "greenization". After the workpiece being carried or moved to the named position, the gear motor is access to the reverse power supply. So the worm will rotate the upper worm gear clockwise and the upper crankconnecting rod articulated in the upper worm gear will move downward in a straight line; The lower worm will rotate the lower worm gear counterclockwise and the lower crank-connecting rod articulated in the lower worm gear will move upward in a straight line. Thus the workpiece will be loosened.

The upper and the lower electrical-clamping devices which increase the force by secondary tandem are arranged symmetrically to make the upper and lower gripping indenters output the equal and opposite force which is in the same line, which can ensure the balance of the workpiece. So the time of gripping and adjusting the position of the workpiece is shorten and the efficiency of gripping workpiece is improved, which can achieve the aim of saving labor and time, and being efficient.

It's necessary for the device to have the self-locking function when the internal-gripping device is designed. There are two kinds of self-locking ways which are commonly used currently; one is based on the principle that the driving force is smaller than the friction force or the driving torque is smaller than the friction torque, such as inclined-wedge, spiral, cam, etc. The other is based on the principle of no dead point [11]. The essence of the worm and worm gear mechanism is spiral mechanism. So a single-start worm should be used and an appropriate lead angle should also be chosen to ensure the reliable of selflock. The lead angle is less than 3.5 degrees normally [12]. The smaller the lead angle is, the more stable the self-lock, the more distinct the reinforcing effect and meanwhile the lower the transmission efficiency and the gripping speed.

The crank-connecting rod mechanism which is in tandem with the worm gear is to transform the rotary motion of the worm gear to the rectilinear motion needed when holding the workpiece tightly. The crank-connecting rod mechanism is a force-amplifier based on angle effect [13] and its output force and displacement have the characteristics of nonlinear variation. That is to say, the gripping indenter has the characteristic of long stroke length and fast speed when the angle is relatively larger. When the angle decreases, the stroke of the gripping indenter becomes relatively smaller and the output force increases rapidly to output great gripping force.

The mechanical calculation of the internal-gripping device of the manipulator

If the torque inputted by motor is $T_{1}$, the output forces of the upper and lower gripping indenters are

$$
\frac{F_{\mathrm{o}}}{n}=\frac{T_{1}}{r \cdot(\tan \alpha \cos \beta+\sin \beta)} \cdot \frac{z_{2}}{z_{1}} \cdot \eta_{1} \cdot \eta_{2}
$$

In the formula, $\mathrm{n}$ is the number of the electricalclamping device which increases the force by secondary tandem; $Z_{2}$ is the number of teeth of the worm gear; the $z_{1}$ is the number of threads of the worm; $r$ is the distance from the center of the worm gear to the hinge joint of the connecting rod; $\alpha$ is the pressure angle of the crank-connecting rod mechanism and $\beta$ is the driving angle of the crank-connecting rod mechanism; $\eta_{1}$ is the transmission efficiency of the worm and worm gear; $\eta_{2}$ is the transmission efficiency between the crank-connecting rod and gripping indenter. When the rotating speed is relatively slower, $\eta_{1}$ is influenced mainly by the meshing efficiency of the worm gear pair, its calculation formula is

$$
\eta_{1}=\frac{\tan \gamma}{\tan (\gamma+\varphi)}
$$

In the formula, $\gamma$ is the lead angle of reference cylinder $\left(^{\circ}\right), \varphi$ is the meshing frictional angle $\left(^{\circ}\right)$ and is determined by $\mu$ which is the meshing friction coefficient, that is to say $\varphi=\arctan \mu$.

$\eta_{2}$ is influenced mainly by the he transmission efficiency between the crank-connecting rod and gripping indenter, its calculation formula is

$$
\eta_{2} \approx 1-\frac{f r \lambda}{r \sqrt{1-\lambda^{2}}}
$$


In the formula, $f$ is rotary vice equivalent friction coefficient, $\lambda$ is the crank rod ratio and is determined by

$\lambda=\frac{r}{1} \pi 1$.

When $\mathrm{n}=2$, the gripping device may grab the Ushaped workpiece, the worm number of gripping device is two and the angle of two worm wheel is $180^{\circ}$ and each other is arranged symmetrically; when $\mathrm{n}=3$, the workpiece for gripping may be a cylindrical crawl, the worm number of gripping means 3 , the angle of 3 worm wheels is $120^{\circ}$ to each other and arranged along the circumferential surface of the worm, which is fixed to the cylindrical workpiece to achieve three-point support; when $\mathrm{n}=4$, the gripping device can grab the square shaped hole shape workpiece, then the worm number is 4 , and the angle between the four worm is $90^{\circ}$ and setted along the worm week face, so it can be fixed on larger workpieces to implement a four-point support; thereby expanding the application scope and the fixed effects of this gripping device.

\section{CONCLUSION}

1) The internal-gripping device which increases the force by connecting the worm and worm gear and the crank-connecting rod tandem can acquire high force increase ratio and has an obvious effect on increasing the force. Meanwhile, the output force and displacement of the crank-connecting rod mechanism have the characteristic of being nonlinear; so the feed of gripping indenter is fast when the pressure angle is big and the gripping force increases rapidly when the pressure angle is small.

2) The gripping force of the gripping state can remain unchanged without any additional energy input by using the self-locking principle of the worm and worm gear mechanism; so the operation is reliable and the structure is simple. Besides, the device does not exist any environmental pollution and has a high degree of greenization.

3) The upper and the lower gripping indenters can move inwards or outwards meanwhile by the symmetrical arrangement of the worm and worm gear and the crankconnecting rod mechanism, which can realize the purpose of finding balance automatically.

4) This internal-gripping device has compact structure, be easy to use, can locate and grab quickly, and can grip the workpiece with different shapes, can grab at multipoint, which expand the use of the device.

The internal-gripping device can be installed on manufacturing manipulator to realize gripping and placing products automatically in automatic production line. The internal-gripping device can also be installed on carrying manipulator to grip or place the carried objects conveniently and timely. Not only can the internalgripping device of the manipulator grip and place workpiece instead of hands but also surpass person's physical fitness. It even can keep working for 24 hours, which can not only reduce the cost of manual work, but also reduce the risk of accidents due to fatigue caused by artificial and so on.

Meanwhile, the device can bear the operation in all kinds of severe environment such as volume-produce, repetitive operation, heavy workpiece, high temperature, dusty, radiation or acid and base condition. The rational use of the device can improve labor productivity and the quality of the product. Besides, it can also reduce the labor intensity of workers and the consumption of energy.

\section{ACKNOWLEDGMENT}

Supported by National Natural Science Foundation of China 51205266, Suzhou Municipal Scientific Program of China SYG201326, and Jiangsu Planned Projects for Postdoctoral Research Funds 1101121C.

\section{REFERENCES}

[1] Li Liang, Snatch target object by manipulator, Chengdu: Xihua University, 2009.

[2] Zhou Hongbo, Design of Deburring Robot System and Its Research on Recognition Technique of Hole Position on Hole Outside a Screen Pipe, Shenyang: Shenyang University of Technology, 2007.

[3] Ju Hongling, Ji Changying, "Design of an end-effector for a multiuse picking robot", Acta Agriculturae Zhejiangensis, vol.3,2010,pp.370-373.

[4] Jing Jianghao, Deng Xiaozhong, Zhang Hua, "Rapid Variant Design and Analysis on an End Effector for Industrial Robot". Machinery Design \& Manufacture, vol.1,2014,pp.157-160.

[5] Bai Ping, Xue Tong, "The Mechanical Design Process of the CNC Machine' s Load\&unload", Public Communication of Science \& Technology, vol.11,2012,pp.71-71.

[6] Shen Dongdong, The Load\&unload of the Machine Tool[EB/OL], http://www.robot-china.com/news/201307/15/4921.html, 2013-715 .

[7] Bai Yinzhuo, Li Xin, Zhong Kangmin, “Two-step Manual Clamping Device Based on Wormgear and Crankshaft In Series", Machinery, vol.4, 2011,pp.69-70.

[8] Wu Xuewei, "The 3-D model and Dynamic simulation of the worm drive", Machinist Metal Cutting, vol.20,2009,pp.72-73.

[9] Wang Yichao, Long Xianjing, "Dynamic Analysis of Crankconnecting Rod of Emulsion Pump", Coal Mine Machinery, vol.6,2010,pp.85-87.

[10] Liu Lili, Study on Green Clamping System Driven by Gear Motor, Suzhou: Soochow University, 2011.

[11] Sun Heng, Chen Zuomo, Ge Wenjie, "Theory of Machines and Mechanisms(The seventh edition)", Beijing: Higher Education Press, 2006.

[12] Wang Zhixing, Deng Zongquan, "Theory of Machines and Mechanisms(The second edition)", Beijing: Higher Education Press, 2008.

[13] Chen Zhisong, The Innovative Design and Mechanical Properties Research of Symmetrical Force-Amplify Crank-Type Presses, Suzhou: Soochow University, 2009. 